\title{
Fighting against a misconception about the Energy Yielding Metabolism: a proposal for starting the teaching of human nutrition in schools
}

\author{
$\underline{F}$ Souza Silva $^{1}$, AT Da Poian ${ }^{1}$ and MRMP Luz $z^{2}, 3$ \\ ${ }^{1}$ Instituto de Bioquímica Médica, UFRJ, ${ }^{2}$ Colégio de Aplicação, UFRJ, ${ }^{3}$ Setor de Inovações Educacionais, \\ Fiocruz
}

We have demonstrated that undergraduate and high school students share two contradictory conceptions regarding the energy yielding metabolism (EYM): the incorrect notion that only glucose can be used for ATP production together with more appropriated conception according to which other molecules, mainly lipids can also be used for ATP production. We have deepened the study about the origin of such conceptual profile. Using questionnaires, we have determined that $7^{\text {th }}$ grade school students do not present the erroneous conception, but those from the $8^{\text {th }}$ grade (and later grades) do. This finding has led us to propose the hypothesis that the wrong conception may emerge as a result of formal learning during $8^{t} h$ grade. Indeed, the analysis of $8^{\text {th }}$ grade textbooks showed that the carbohydrates were associated mainly with energy production by the cells, while proteins and lipids were regarded only as structural and storage molecules, respectively. In addition, only the glycolytic pathway was taught in high school, reinforcing the misconception. We have also demonstrated that the conceptual profile was widely distributed among students from different schools of different regions of the Rio de Janeiro state, reinforcing the importance of reformulation of the teaching of EYM-related topics both in grade and high schools. Here we describe the development and the evaluation of an investigation-based approach to human nutrition to be used with $8^{t} h$ grade students. This methodology is based in the use of the nutritional tables found in Brazilian food packages and allows the students to identify not only which of the main nutrients do contain calories (and thus can be used for "energy production") but the amount of calories found in $1 \mathrm{~g}$ of each of them. The methodology was tested in different schools and the results show that the majority of the students become aware that carbohydrates, proteins and lipids contain calories. The methodology described in association with a more integrated teaching of the EYM in high schools may prevent or at least reduce the occurrence of misconceptions among students. Supported by CNPq and FAPERJ. 